\title{
Pediatric lymphoma patients in Malawi present with poor health-related quality of life at diagnosis, and improve throughout treatment and follow-up across all Pediatric PROMIS-25 domains.
}

\author{
Grace Ellis ${ }^{1}$, Hutton Chapman ${ }^{2}$, Agness Manda ${ }^{1}$, Ande Salima ${ }^{3}$, Salama Itimu ${ }^{1}$, Grace \\ Banda $^{1}$, Ryan Seguin ${ }^{1}$, Geoffrey Manda ${ }^{4}$, Mercy Butia ${ }^{4}$, Minke Huibers ${ }^{4}$, Nmazuo Ozuah $^{4}$, \\ Alyssa Tilly ${ }^{2}$, Angela Stover ${ }^{5}$, Ethan Basch ${ }^{2}$, Satish Gopal ${ }^{6}$, Bryce Reeve ${ }^{7}$, and Kate \\ Westmoreland ${ }^{2}$ \\ ${ }^{1}$ UNC Project \\ ${ }^{2}$ University of North Carolina at Chapel Hill School of Medicine \\ ${ }^{3}$ University of North Carolina Project \\ 4Texas Children's Hospital \\ ${ }^{5}$ University of North Carolina at Chapel Hill Gillings School of Global Public Health \\ ${ }^{6}$ University of North Carolina at Chapel Hill Department of Medicine \\ ${ }^{7}$ Duke University School of Medicine
}

November 10, 2020

\begin{abstract}
Background: Patient-reported outcomes (PROs) that assess health-related quality of life (HRQoL) are increasingly important components of cancer care and research that have been infrequently used in sub-Saharan Africa (SSA). We aimed to longitudinally measure HRQoL among pediatric lymphoma patients in Malawi. Methods: We administered the Chichewa Pediatric Patient-Reported Outcome Measurement Information System Pediatric (PROMIS)-25 at diagnosis, active treatment, and follow-up among pediatric lymphoma patients in Lilongwe, Malawi. Mean scores were calculated for the six PROMIS-25 HRQoL domains (Mobility, Anxiety, Depressive Symptoms, Fatigue, Peer Relationships, and Pain Interference) using the PROMIS scoring manual. Results: Seventy-five children completed PROMIS-25 surveys at diagnosis, 35 (47\%) during active treatment, and $24(32 \%)$ at follow-up. The majority of patients died $(\mathrm{n}=37,49 \%)$ or were lost-to-follow-up $(\mathrm{n}=8,11 \%)$. Most $(\mathrm{n}=51,68 \%)$ were male, median age was 10 (IQR 8-12), 48 (66\%) presented with advanced Stage III/IV, 61 (81\%) were diagnosed with Burkitt lymphoma and 14 (19\%) had Hodgkin lymphoma. At diagnosis, HRQoL was poor across all domains, except for Peer Relationships. Improvements in HRQoL during active treatment and follow-up exceeded the minimally important difference. Poor Lanksy performance status [?] 70 and Pain Intensity $=10$ at diagnosis were associated with increased mortality risk and worse survival. Conclusions: Our experience suggests incorporating assessments of HRQoL via PROs in oncology care is feasible in SSA, can provide prognostic information, and generates clinically meaningful data to inform supportive care interventions. Further, PROs offer an opportunity to include patient voices and prioritize holistic patient-centered care even in low-resource settings.
\end{abstract}

Title: Pediatric lymphoma patients in Malawi present with poor health-related quality of life at diagnosis and improve throughout treatment and follow-up across all Pediatric PROMIS-25 domains

Running Title : Quality of life during lymphoma treatment in Malawi

Authors: Grace K. Ellis, ${ }^{1}$ Hutton Chapman, ${ }^{2}$ Agness Manda, ${ }^{1}$ Ande Salima, ${ }^{1}$ Salama Itimu, ${ }^{1,3}$ Grace 
Banda, ${ }^{1}$ Ryan Seguin, ${ }^{1}$ Geoffrey Manda,${ }^{3}$ Mercy Butia, ${ }^{3}$ Minke Huibers,${ }^{3,4}$ Nmazuo Ozuah, ${ }^{3,4}$ Alyssa Tilly, ${ }^{2}$ Angela M. Stover, ${ }^{2}$ Ethan Basch, ${ }^{2}$ Satish Gopal, ${ }^{1,2}$ Bryce B. Reeve, ${ }^{5}$ Katherine D. Westmoreland. ${ }^{1,2}$

Affiliations: ${ }^{1}$ UNC Project-Malawi, ${ }^{2}$ University of North Carolina at Chapel Hill, ${ }^{3}$ Texas Children's Cancer and Hematology Centers, Global Hematology-Oncology Pediatric Excellence (HOPE) Program, ${ }^{4}$ Baylor College of Medicine, ${ }^{5}$ Duke University School of Medicine

\section{Corresponding Author:}

Dr. Kate Westmoreland

Email: kawestmo@email.unc.edu

US Mobile: +1 (336) 414-8811, Malawi Mobile: +265 996466 771, Fax: +1 (919) 966-7629

USA Address: 170 Manning Drive, Campus Box 7236, Chapel Hill, NC 27599

Malawi Address: UNC Project Malawi, Private Bag 104A, Lilongwe, Malawi

Keywords: health-related quality of life, patient-reported outcomes, pediatric oncology, sub-Saharan Africa, Burkitt lymphoma, Hodgkin Lymphoma

Word count: Abstract 250 (of 250); Body 3,227 (of 3,500)

Tables \& Figures:

Tables - 2; Figures - 4; Supplemental Table - 1; Supplemental Figure - 1

Abbreviations :

\begin{tabular}{ll}
\hline PROs & patient-reported outcomes \\
\hline HRQoL & health-related quality of life \\
SSA & sub-Saharan Africa \\
PROMIS & Patient-Reported Outcome Measurement Information System Pediatric \\
LMICs & low- and middle-income countries \\
HICs & high-income countries \\
KCH & Kamuzu Central Hospital \\
UNC & University of North Carolina \\
HOPE & Hematology-Oncology Pediatric Excellence \\
LPS & Lansky performance score \\
LTFU & lost to follow-up \\
MID & minimally important difference \\
LDH & lactate dehydrogenase \\
ULN & upper limit of normal \\
SD & standard deviation \\
IQR & interquartile range \\
\hline
\end{tabular}

Abstract

Background: Patient-reported outcomes (PROs) that assess health-related quality of life (HRQoL) are increasingly important components of cancer care and research that have been infrequently used in subSaharan Africa (SSA). We aimed to longitudinally measure HRQoL among pediatric lymphoma patients in Malawi.

Methods: We administered the Chichewa Pediatric Patient-Reported Outcome Measurement Information System Pediatric (PROMIS)-25 at diagnosis, active treatment, and follow-up among pediatric lymphoma patients in Lilongwe, Malawi. Mean scores were calculated for the six PROMIS-25 HRQoL domains (Mobility, 
Anxiety, Depressive Symptoms, Fatigue, Peer Relationships, and Pain Interference) using the PROMIS scoring manual.

Results: Seventy-five children completed PROMIS-25 surveys at diagnosis, 35 (47\%) during active treatment, and $24(32 \%)$ at follow-up. The majority of patients died $(\mathrm{n}=37,49 \%)$ or were lost-to-follow-up $(\mathrm{n}=8$, $11 \%)$. Most ( $\mathrm{n}=51,68 \%)$ were male, median age was 10 (IQR 8-12), 48 (66\%) presented with advanced Stage III/IV, 61 (81\%) were diagnosed with Burkitt lymphoma and 14 (19\%) had Hodgkin lymphoma. At diagnosis, HRQoL was poor across all domains, except for Peer Relationships. Improvements in HRQoL during active treatment and follow-up exceeded the minimally important difference. Poor Lanksy performance status [?] 70 and Pain Intensity $=10$ at diagnosis were associated with increased mortality risk and worse survival.

Conclusions: Our experience suggests incorporating assessments of HRQoL via PROs in oncology care is feasible in SSA, can provide prognostic information, and generates clinically meaningful data to inform supportive care interventions. Further, PROs offer an opportunity to include patient voices and prioritize holistic patient-centered care even in low-resource settings.

\section{Introduction}

Children and adolescents with cancer in low- and middle-income countries (LMICs) have poor outcomes and improving their care is a significant global health priority. ${ }^{1}$ Five-year survival rates for children with cancer in high-income countries (HICs) are approximately $80 \%$, but are estimated to be only $30 \%$ in LMICs. ${ }^{1-4}$ In sub-Saharan Africa (SSA), pediatric cancer experiences are often marked by high rates of treatment failure, due to poor treatment access, high treatment abandonment, and high treatment-related mortality. ${ }^{1,2,4-6}$ Additionally, palliative and supportive care capacity is limited for children with cancer in SSA. ${ }^{4}$ Ongoing efforts in SSA to improve survival rates include numerous initiatives to identify and implement contextspecific adaptions of standard HIC approaches. ${ }^{1,4,7-9}$

Patient-reported outcomes (PROs) offer an important opportunity to incorporate patients' voices and experiences in evidenced-based interventions across SSA. PROs have become a routine part of oncologic care in many HICs and measure how mental, physical, and social health-related quality of life (HRQoL) are affected by cancer diagnosis, treatment, and survivorship. PROs provide critical information to complement clinical assessments, monitor disease- and treatment-related adverse events, and guide symptom management. ${ }^{10-16}$ Standardized instruments for self-reporting HRQoL domains were created by the United States National Institutes of Health Patient-Reported Outcomes Measurement Information System ${ }^{\circledR}\left(\right.$ PROMIS $\left.{ }^{\circledR}\right)$ initiative. Patient-reported HRQoL measures are a more sensitive measure of functional status than clinician-reported assessments, and incorporating PROs into cancer treatment can improve outcomes and survival. ${ }^{14,16}$ Despite their utility and frequent use in HICs, PROs are rarely used in SSA.

We hypothesized that patient-reported HRQoL would have particular utility in Malawi, where curativeintent treatments are often limited, advanced stage diagnoses are common, the risk for treatment-related toxicity is high, and supportive care infrastructure is often insufficient. The Pediatric PROMIS measures have been validated among pediatric and adolescent cancer patients in the United States. ${ }^{17-19}$ In prior work, our group has translated and validated the Pediatric PROMIS-25 questionnaire into Chichewa, Malawi's national language, which was the first Pediatric PROMIS translation and validation into a Bantu language for use in SSA. ${ }^{20}$ The Pediatric PROMIS-25 is a short form composed of 25 questions assessing mental, physical, and social components of HRQoL. We measured HRQoL among pediatric lymphoma patients using the Chichewa Pediatric PROMIS-25 instrument at diagnosis, and found our patients had poor HRQoL at diagnosis compared to the PROMIS pediatric reference population of children with cancer in the United States. ${ }^{20}$ We extend our prior observations to describe longitudinal changes in HRQoL from diagnosis through active treatment and follow-up, to demonstrate the feasibility of using PROs in SSA, and to explore if PROMIS measures can be predictive of survival.

\section{Methods}




\section{Setting}

Kamuzu Central Hospital $(\mathrm{KCH})$ is a national teaching hospital in Lilongwe, the capital of Malawi, and is one of two hospitals in the country that provides pediatric cancer treatment. $\mathrm{KCH}$ receives cancer referrals from the northern and central regions, serving half of the estimated 18 million people in Malawi. The $\mathrm{KCH}$ pediatric cancer ward consists of 24-30 beds and is staffed by the Texas Children's Cancer and Hematology Centers Global Hematology Oncology Pediatric Excellence (HOPE)-Malawi program. Collaboration with the University of North Carolina (UNC) Project-Malawi provides additional support including a pediatric hematologist-oncologist, clinical research staff, comprehensive diagnostic services through the KCH-UNC Pathology Laboratory. ${ }^{21}$

\section{Participants}

We implemented the Chichewa Pediatric PROMIS-25 measure to assess HRQoL of patients aged 5-18 years with lymphoma who were diagnosed at KCH between June 2016 and September 2018. All patients were enrolled in the KCH Lymphoma study, an observational prospective cohort study that began in 2013, and were followed for two years after treatment completion. ${ }^{2,22,23}$

Patients were staged using physical examination, chest radiography, abdominal ultrasound, unilateral bone marrow biopsy, and cerebrospinal fluid cytology, as previously described. ${ }^{2,22}$ The clinician-reported Lansky performance score (LPS), routinely used in cancer clinical trials for children $<16$ years, was obtained at diagnosis. ${ }^{24}$ All lymphoma diagnoses were pathologically confirmed using tissue biopsies and immunohistochemistry and were interpreted during real-time weekly telepathology conferences. ${ }^{21}$

Standard chemotherapy regimens were administered and evolved during the study period, and as previously described: Hodgkin lymphoma patients received ABVE-PC regimen (doxorubicin, bleomycin, vincristine, etoposide - prednisone \& cyclophosphamide); low-risk Burkitt Lymphoma patients received COMP (cyclophosphamide, vincristine, methotrexate and prednisone); and high-risk Burkitt lymphoma initially received CHOP (cyclophosphamide, doxorubicin, vincristine, prednisone) and more recently received modified COPADM (cyclophosphamide, vincristine, prednisone, doxorubicin, methotrexate). ${ }^{2,22,23}$ Radiotherapy is not available in Malawi.

\section{Study Design and Procedures}

We previously reported Chichewa translation and validation of the Pediatric PROMIS-25 survey. ${ }^{20}$ The Chichewa Pediatric PROMIS-25 short form was administered at diagnosis (at the time of study enrollment prior to initiation of chemotherapy), during active treatment (within 30 days of last receiving chemotherapy, typically on day 0 of the next scheduled chemotherapy) and at follow-up (patients returning for a scheduled follow-up clinic visit between 6 months and 2 years after treatment completion). If patients progressed or relapsed and were receiving second line chemotherapy at the time of the PROMIS-25 administration, then this was categorized as active treatment. If they progressed or relapsed, but were on palliative care and not receiving chemotherapy, then this was categorized as follow-up.

For children [?]8 years old, the Chichewa Pediatric PROMIS-25 was administered directly to the child. The parent-proxy version was used for children 5-7 years old, cognitively impaired, or too ill to complete the questionnaire themselves. ${ }^{20,25-27}$ Questionnaires were read aloud to the child/parent via a non-provider research assistant trained in survey methodology and recorded on a paper form. This modification was deemed necessary in our setting due to low literacy in the target population. The parent/child had an answer choice sheet in front of them so they could point to choose their answers, a method previously used by our team in Malawi and validated for other PRO-HRQoL instruments targeting young children. ${ }^{20,28}$

After treatment completion, some patients were lost to follow-up (LTFU) from clinic but reachable by phone. We encouraged these patients to return to clinic in person, and when unsuccessful we administered the PROMIS-25 survey via phone. Prior work has demonstrated that in person and telephone administration of English Pediatric PROMIS measure does not affect validity, although this has not been demonstrated for the Chichewa version. ${ }^{29,30}$ 


\section{Analysis}

The Pediatric PROMIS-25 measured six HRQoL domains (Mobility, Anxiety, Depressive Symptoms, Fatigue, Peer Relationships, Pain Interference) by asking four questions per domain using a five-point Likert scale (e.g.,never, almost never, sometimes, often, almost always ; or, with no trouble, with a little trouble, with some trouble, with a lot of trouble, not able to ). Additionally, a single-item PROMIS Pain Intensity item was measured on a scale of 0 to 10 . All items have a 7-day recall period.

Within each domain, a raw score was calculated as the sum of the answers to the four question items. Raw scores were transposed to standardized domain T-scores using tables provided by the PROMIS scoring manual, with a mean of 50 and standard deviation of $10 .{ }^{31}$ The PROMIS standardized T-score is composed from a mixed calibration sample of United States children in good health and children with a wide range of chronic conditions. ${ }^{32}$ Higher PROMIS scores for Mobility and Peer Relationships reflect better functioning and relationships, with scores $<40$ (1 standard deviation (SD) below the mean) considered fair to poor. ${ }^{33}$ Higher symptom scores reflect worse symptom experiences (Anxiety, Depressive Symptoms, Fatigue, Pain Interference), with scores $>55$ (1 SD above the mean) indicating moderate to severe symptom burden. ${ }^{33}$

Differences between diagnosis, active treatment, and follow-up domain mean T-scores were compared to published minimally important difference (MID) estimates of 2-3 points, and also analyzed using an ANOVA test. ${ }^{34}$ For sensitivity analyses, parent proxy-reported surveys were removed, as it has been demonstrated that caregivers consistently overestimate symptoms and underestimate function relative to child self-reports. ${ }^{35}$

Cohort characteristics were summarized using simple descriptive statistics. Patients were followed from enrollment until death, LTFU, or administrative censoring on January 31, 2020. We conducted an exploratory analysis to compare outcomes among patients with outcomes for those with poor versus adequate HRQoL domain scores. Kaplan-Meier methods were used to estimate overall survival and risk factors for mortality were assessed using univariate Cox proportional-hazards regression analysis to estimate hazard ratios (HR) of quantitative predictor variables. Analyses were conducted using R 3.5.2 (New York, New York).

Ethical Approval

All participants gave written informed consent by their legal guardian, and children [?] 7 years also provided pediatric assent. This study was approved by the UNC Institutional Review Board and Malawi National Health Sciences Research Committee.

\section{Results}

Seventy-five children had Chichewa Pediatric PROMIS-25 surveys administered at diagnosis prior to initiation of chemotherapy, 35 (47\%) during active treatment, and $24(32 \%)$ at follow-up (Figure 1, Table 2). Median age was 10 years (Interquartile range (IQR) 8-12) and 51 (68\%) were male. Sixty-one (81\%) patients were diagnosed with Burkitt lymphoma and 14 (19\%) with Hodgkin lymphoma. Most patients presented with stage III/IV disease $(\mathrm{n}=48,66 \%)$, poor LPS of [?] $70(\mathrm{n}=50,70 \%)$, and moderate-to-severe acute malnutrition ( $\mathrm{n}=54,72 \%)$. Median lactate dehydrogenase (LDH) was 555 IU/L (IQR 378-895), with 30 (40\%) $>2 \mathrm{x}$ LDH upper limit of normal for their age (Table 1 ).

Of the 75 surveys at diagnosis (Figure 1), patients reported poor HRQoL, with low Mobility and high Anxiety, Depressive Symptoms, Fatigue, and Pain Interference mean domain scores (Table 1, Table 2, Figure $3)$. Patients also presented with high pain intensity, with $32(43 \%)$ reporting the maximum Pain Intensity of 10 on a scale of 0 to 10 at diagnosis (Table 1, Table 2). Peer Relationships were strong at diagnosis (Table 1 , Table 2).

Of the 35 surveys during active treatment (Figure 1), the median time of survey administration was 113 days (IQR 61-210) after diagnosis (Table 2). Mean HRQoL domain scores significantly improved in all domains $(\mathrm{p}<0.001)$ (Table 2), and differences between diagnosis and active treatment domain scores exceeded accepted MID thresholds (Table 2, Figure 3). Pain Intensity decreased from a median of 9 (IQR 4-10) at diagnosis to a median of 1 (IQR 1-1) during active treatment (Table 2). 
Of the 24 follow-up surveys (Figure 1), two patients (14\%) had relapsed lymphoma and were receiving palliative care during follow-up. Median follow-up time was 720 days (IQR 486-1014) since diagnosis (Table 2). All mean HRQoL domains showed continued improvement from diagnosis to active treatment to followup timepoints that surpassed the MID (Table 2, Figure 3). Notably, median Pain Intensity score was 0 (IQR $0-0$ ) at follow-up (Table 2).

Of 134 total Pediatric PROMIS-25 surveys administered across the 3 time points, 100/134 (75\%) were to patients and $34 / 134(25 \%)$ were to parents. Of the 34 surveys completed by parent proxy, $14 / 34(41 \%)$ were for children younger than 8 years, 20/34 (59\%) for older children who were too ill to complete the survey on their own. Improvements in HRQoL remained when proxy reports were removed from the analysis (Supplemental Table 1). Almost all surveys (125/134, 93\%) were conducted in person, aside from 9/134 (7\%) follow-up surveys administered over the phone.

Of the 75 patients enrolled at diagnosis, 37 (49\%) of patients died-34/37 (92\%) Burkitt, 3/37 (8\%) Hodgkin - and 8 (11\%) were LTFU (Figure 1). Kaplan-Meier overall survival was 47\% (95\% CI [37-59\%]) at 1-year and 38\% (95\% CI [28-51\%]) at 2-years. Some patient characteristics at diagnosis significantly differed when stratified based on survival outcome (alive vs. LTFU/died) including: diagnosis (Burkitt lymphoma versus Hodgkin lymphoma), Lansky performance score [?] 70, median LDH, and mean Mobility domain scores (Table 1). Median Pain Intensity scores at diagnosis were also poorer among patients who died/LTFU compared to those who survived (10.0, IQR 6.0-10.0 vs. 8.5, IQR 3.3-10.0; $\mathrm{p}=0.073$ ) (Table 1).

On exploratory analysis, patients with poor LPS [?] 70 at diagnosis had an increased mortality risk (Hazard ratio $(\mathrm{HR})=5.20,95 \%$ CI $[1.35-6.91], \mathrm{p}=0.007)$ and worse overall survival compared to those with LPS $>70(35 \%, 95 \%$ CI [24-51\%] vs. $76 \%, 95 \%$ CI [60-97\%], p=0.0049) (Figure 2A). Likewise, patients with the highest Pain Intensity score of 10 at diagnosis also had increased mortality risk $(\mathrm{HR}=1.75,95 \%$ CI [0.97-3.15], $\mathrm{p}=0.06)$ and worse survival compared to those with a Pain Intensity score $<10(36 \%, 95 \%$ CI [23-58\%] vs. $56 \%, 95 \%$ CI [43-73\%], p=0.058) (Figure 2B). A poor Mobility domain score $<40$ at diagnosis was associated with increased mortality risk $(\mathrm{HR}=2.05,95 \% \mathrm{CI}[0.73-5.73], \mathrm{p}=0.17)$ and worse survival compared to those with Mobility scores [?] 40 (44\%, 95\% CI [33-58\%] vs. 60\%, 95\% CI [36-100\%], p=0.16) (Supplemental Figure 1). Although clinically meaningful, this was not statistically significant.

Domain score trajectories were plotted for the 17 patients who completed PROMIS surveys at all three time points (diagnosis, active treatment, and follow-up) to visualize individual changes in HRQoL throughout care (Figure 4). This sub-analysis allows interpretation of longitudinal HRQoL trends in our cohort without potential effects of survivor bias. Overall, HRQoL improvements were observed for most patients $(\mathrm{n}=15$, $88 \%)$, with the exception of two patients (12\%) who had relapsed disease and worse HRQoL at follow-up.

\section{Discussion}

We report the first longitudinal assessment of HRQoL using PROs in SSA among pediatric cancer patients. We used the Chichewa Pediatric PROMIS-25 to serially measure HRQoL among pediatric lymphoma patients in Malawi. Our experience highlights important disparities in pediatric cancer care experiences and suggests incorporating assessments of HRQoL via PROs in SSA is feasible, generates clinically meaningful data to inform supportive care interventions, and may provide prognostic information.

Our cohort presented with overwhelmingly advanced disease, exemplified by late stage at diagnosis, high $\mathrm{LDH}$, and poor performance status, which is common across SSA. ${ }^{2,36}$ We have previously demonstrated that HRQoL at diagnosis is poorer among Malawian patients than the PROMIS reference population and similar pediatric oncology populations in the United States, likely reflecting more advanced disease. ${ }^{32}$ In HICs, children with cancer are frequently diagnosed early in their disease course with no/minimal symptom burden and have preserved HRQoL. ${ }^{17,37}$ In contrast, our patients reported poor HRQoL and extremely high pain intensity, with $32 / 75$ (43\%) of patients reporting a maximum 10/10 pain score at diagnosis. Pain medication is available in Malawi, but the extreme pain burden among our patients suggests their pain may not have been identified or adequately managed prior to referral and/or diagnosis. Efforts to educate providers on appropriate pain management in children and ensure reliable medication access, as well as 
outreach to facilitate early cancer diagnosis and referral are priorities. ${ }^{1,38-40}$

Patients reported significantly improved HRQoL during active treatment that far exceeded ( $>12$ points) the MID thresholds of 2-3 points, with the exception of peer relationships which improved by 3.3 points. These improvements likely reflect reduced tumor burden and improved symptom management during active treatment. HRQoL continued to improve after treatment completion and through follow-up. In contrast, children with cancer in HICs have no/ minimal symptom burden at diagnosis, and report decreased HRQoL during cancer treatment due to therapy-related adverse effects, which then improves following treatment completion. ${ }^{17,37}$

One unique observation was that peer relationships were strong throughout all timepoints. Patients in our cohort often receive treatment in an open pediatric ward and are able to develop strong friendships with other children undergoing similar experiences. Additionally, they remain socially connected in their home villages, where they live in close proximity with their neighbors, family, and friends, and continue to attend school during their treatment course. The preservation of peer relationships throughout cancer treatment in Malawi is likely common in other LMICs but is not observed among pediatric cancer patients in HIC. In HIC, children with cancer are typically treated in private rooms and with infection control restrictions, thus experiencing significant decreases in social HRQoL with poor peer relationship scores during treatment. ${ }^{17,18,20,41,42}$

We are sad to report that mortality in our cohort was high. Most of our patients died $(37 / 75,49 \%)$ or were LTFU $(8 / 75,11 \%)$, an indication of unverifiable death in our setting. ${ }^{43}$ One-year overall survival was $47 \%$ (95\% CI [37-59\%]), which is consistent with historical and published survival rates for Burkitt lymphoma across SSA, and is significantly worse than reported survival rates of $>90 \%$ in HIC. ${ }^{2,22,43-47}$ Given the high mortality and the challenges associated with cancer treatment in resource-poor health systems, appropriate risk stratification of patients is critical in SSA, where administering aggressive treatment to optimize survival must be weighed against toxicity risks for vulnerable children. Poor LPS has consistently been identified as a risk factor for mortality in research from both LMICs and HICs. ${ }^{2,22,45,46,48}$ Further, emerging data from HICs suggest PROs offer superior assessment of performance status when compared to traditional physician-reported measures. ${ }^{49}$ We observed significantly increased mortality risk and worse survival in our cohort among patients with physician-reported LPS [?] 70 and patient-reported Pain Intensity of 10 at diagnosis. Patient-reported poor Mobility domain scores $<40$ also increased risk of mortality and worse overall survival, but this trend was not statically significant. Further evaluation of the potential prognostic utility of Pediatric PROMIS-25 in a larger patient cohort with adequate power is necessary to understand how PROs can assist with treatment risk stratification in SSA.

PROs are especially important in SSA, where providers are often over-worked, healthcare infrastructure/access is limited, and shared decision making between patients and providers is not the norm. Assessments of HRQoL via PROMIS can assist providers in quickly identifying and prioritizing patients for referral to ancillary, multidisciplinary providers, such as physiotherapists and palliative/supportive care specialists. Further, PROMIS measures mental wellbeing, which is often overlooked in pediatric oncology despite being a significant problem, and can screen patients for anxiety and depression to facilitate referrals for psychosocial support. ${ }^{50}$ In addition to generating clinically meaningful data, Pediatric PROMIS-25 surveys were feasible to administer at clinical visits while patients were receiving chemotherapy infusions or waiting to be seen by the provider.

Study strengths include prospective design, longitudinal data collection, and use of the first rigorously validated Bantu-language pediatric PRO instrument to assess HRQoL among pediatric cancer patients in SSA. Our data are limited by high mortality, which is comparable to other pediatric lymphoma studies across SSA, and LTFU, which is low compared to other studies in the region. ${ }^{2,22,44,51,52}$ This may have introduced survivorship bias in observed HRQoL improvements during active treatment and follow-up. However, among the 17 patients who completed surveys at all three timepoints, we observed substantial improvements across all HRQoL domains, except for two patients with relapsed disease. An additional limitation is the use of parent-proxy reporting for some children $(35 / 140,25 \%)$, as parents tend to report worse HRQoL than children self-report. ${ }^{35}$ However, our sensitivity analysis without parent proxy-reported surveys showed that HRQoL 
mean domain scores did not significantly differ. Finally, raw PROMIS-25 scores were transposed to Tscores based on the Pediatric PROMIS reference population of children in the United States. As PROMIS is increasingly translated and incorporated into care throughout the world, region-specific reference populations will facilitate better interpretation of patient HRQoL scores. In our future work, we are developing voiceenabled tablet-based software to administer PROMIS-25 surveys without an intermediary to allow for a truly patient-reported measure in our low-literacy patient population.

\section{Conclusion}

Children with lymphoma in Malawi present with poor HRQoL at diagnosis that improves during active treatment and follow-up across all PROMIS-25 domains. The Chichewa Pediatric PROMIS-25 is feasible to administer in SSA, generates clinically meaningful data that can inform multidisciplinary supportive care interventions, and may provide useful prognostic information among pediatric cancer patients. The use of translated PROMIS-25 surveys in both research and routine clinical care includes patient voices in decision making, with a focus on mental, physical, and social HRQoL, and can strengthen holistic patient-centered oncology care across SSA.

Acknowledgements: We would like to thank the patients and their families for participating in the project. We are also grateful to the leadership of Kamuzu Central Hospital, Malawi Ministry of Health, Texas Children's Cancer and Hematology Centers Global HOPE Malawi program, UNC Project-Malawi, UNC Institute for Global Health and Infectious Disease, and UNC Lineberger Comprehensive Cancer Center for support of this study.

Funding: This work was supported by National Institute of Health (NIH) (K01TW011191 [KDW], K01TW009488 [SG] and U2GPS001965); by NIH National Cancer Institute (NCI) (U54CA190152 [SG], P30CA016086, UM1CA121947 [KDW]), by an NIH National Institute of General Medical Sciences Award (T32GM086330 [KDW]); and by an NIH Research Training Grant (D43TW009340 [KDW]) funded by the NIH Fogarty International Center, National Institute of Neurological Disorders and Stroke, National Institute of Mental Health, National Heart, Lung, and Blood Institute, and National Institute of Environmental Health Sciences.

The funding agencies had no role in study design, data collection, data analysis, data interpretation, writing of the report, or the decision to submit it for publication. The content is solely the responsibility of the authors and does not necessarily represent the official views of the NIH.

Conflict-of-interest disclosure: The authors declare no competing financial interests.

Author Contributions: KDW designed the study and provided oversight and mentorship throughout the project. GKE analyzed and interpreted the data with assistance from KDW and BBR. GKE and HC wrote the paper with revisions from KDW, SG, BBR, NO, MH, AMS, AT, GM, EB. AS, SI, GB conducted PROMIS surveys with participants. KDW, NO, MH, GM, MB provided clinical care. RS provided program setup and management.

\section{References}

1. Lam CG, Howard SC, Bouffet E, Pritchard-Jones K. Science and health for all children with cancer. Science . 2019;363(6432):1182-1186. doi:10.1126/science.aaw4892

2. Stanley CC, Westmoreland KD, Heimlich BJ, et al. Outcomes for paediatric Burkitt lymphoma treated with anthracycline-based therapy in Malawi. Br J Haematol . 2016;173(5):705-712. doi:10.1111/bjh.13986

3. Paintsil V, David H, Kambugu J, et al. The Collaborative Wilms Tumour Africa Project; Baseline evaluation of Wilms tumour treatment and outcome in eight institutes in sub-Saharan Africa. Eur J Cancer . 2015;51(1):84-91. doi:10.1016/j.ejca.2014.10.030

4. Magrath I, Steliarova-Foucher E, Epelman S, et al. Paediatric cancer in low-income and middle-income countries. Lancet Oncol . 2013;14(3):e104-16. doi:10.1016/S1470-2045(13)70008-1 
5. Howard SC, Zaidi A, Cao X, et al. The My Child Matters programme: effect of public-private partnerships on paediatric cancer care in low-income and middle-income countries. Lancet Oncol . 2018;19(5):e252-e266. doi:10.1016/S1470-2045(18)30123-2

6. Stanley CC, van der Gronde T, Westmoreland KD, et al. Risk factors and reasons for treatment abandonment among children with lymphoma in Malawi. Support Care Cancer . 2018;26(3):967-973. doi:10.1007/s00520-017-3917-z

7. Khalek ER, Afungchwi GM, Beltagy $\mathrm{M} \mathrm{El}$, et al. Highlights from the 13th African Continental Meeting of the International Society of Paediatric Oncology (SIOP), 6-9 March 2019, Cairo, Egypt.Ecancermedicalscience . 2019;13:932. doi:10.3332/ecancer.2019.932

8. Gopal S, Loehrer PJ. Global Oncology. JAMA - J Am Med Assoc . 2019;322(5):397-398. doi:10.1001/jama.2019.7081

9. WHO | Global Initiative for Childhood Cancer. WHO . 2018.

10. Dudgeon D. The Impact of Measuring Patient-Reported Outcome Measures on Quality of and Access to Palliative Care. J Palliat Med . 2018;21(S1):S76-S80. doi:10.1089/jpm.2017.0447

11. Calvert M, Blazeby J, Altman DG, Revicki DA, Moher D, Brundage MD. Reporting of patient-reported outcomes in randomized trials: The CONSORT PRO extension. JAMA - J Am Med Assoc . 2013;309(8):814822. doi:10.1001/jama.2013.879

12. Banerjee AK, Okun S, Edwards IR, et al. Patient-reported outcome measures in safety event reporting: PROSPER consortium guidance.Drug Saf . 2013;36(12):1129-1149. doi:10.1007/s40264-013-0113-z

13. Mercieca-Bebber R, King MT, Calvert MJ, Stockler MR, Friedlander M. The importance of patientreported outcomes in clinical trials and strategies for future optimization. Patient Relat Outcome Meas . 2018;9:353-367. doi:10.2147/PROM.S156279

14. Basch E. Patient-Reported Outcomes - Harnessing Patients' Voices to Improve Clinical Care. N Engl J Med . 2017;376(2):105-108. doi:10.1056/NEJMp1611252

15. Basch E, Reeve BB, Mitchell SA, et al. Development of the National Cancer Institute's patient-reported outcomes version of the common terminology criteria for adverse events (PRO-CTCAE). J Natl Cancer Inst . 2014;106(9). doi:10.1093/jnci/dju244

16. Basch E. The Missing Voice of Patients in Drug-Safety Reporting. N Engl J Med . 2010;362(10):865-869. doi:10.1056/NEJMp0911494

17. Hinds PS, Nuss SL, Ruccione KS, et al. PROMIS pediatric measures in pediatric oncology: Valid and clinically feasible indicators of patient-reported outcomes. Pediatr Blood Cancer . 2013;60(3):402-408. doi: $10.1002 /$ pbc. 24233

18. Reeve BB, Edwards LJ, Jaeger BC, et al. Assessing responsiveness over time of the PROMIS(r) pediatric symptom and function measures in cancer, nephrotic syndrome, and sickle cell disease. Qual Life Res . 2018;27(1):249-257. doi:10.1007/s11136-017-1697-z

19. Reeve BB, McFatrich M, Mack JW, et al. Expanding construct validity of established and new PROMIS Pediatric measures for children and adolescents receiving cancer treatment. Pediatr Blood Cancer . 2020;67(4):e28160. doi:10.1002/pbc.28160

20. Westmoreland K, Reeve BB, Amuquandoh A, et al. Translation, psychometric validation, and baseline results of the Patient-Reported Outcomes Measurement Information System (PROMIS) pediatric measures to assess health-related quality of life of patients with pediatric lymphoma in Malawi. Pediatr Blood Cancer . 2018;65(11):e27353. doi:10.1002/pbc.27353 
21. Montgomery ND, Liomba NG, Kampani C, et al. Accurate Real-Time Diagnosis of Lymphoproliferative Disorders in Malawi Through Clinicopathologic Teleconferences. Am J Clin Pathol . 2016;146(4):423-430. doi:10.1093/ajcp/aqw118

22. Westmoreland KD, El-Mallawany NK, Kazembe P, Stanley CC, Gopal S. Dissecting heterogeneous outcomes for paediatric Burkitt lymphoma in Malawi after anthracycline-based treatment. $\mathrm{Br} J$ Haematol . 2018;181(6):853-854. doi:10.1111/bjh.14716

23. Westmoreland KD, Stanley CC, Montgomery ND, et al. Hodgkin lymphoma, HIV, and Epstein-Barr virus in Malawi: Longitudinal results from the Kamuzu Central Hospital Lymphoma study. Pediatr Blood Cancer . 2017;64(5). doi:10.1002/pbc.26302

24. Lansky SB, List MA, Lansky LL, Ritter-Sterr C, Miller DR. The measurement of performance in childhood cancer patients. Cancer . 1987;60(7):1651-1656. doi:10.1002/1097-0142(19871001)60:7<1651::aidcncr2820600738>3.0.co;2-j

25. Irwin DE, Gross HE, Stucky BD, et al. Development of six PROMIS pediatrics proxy-report item banks. Health Qual Life Outcomes . 2012;10. doi:10.1186/1477-7525-10-22

26. Varni JW, Thissen D, Stucky BD, et al. PROMIS(r) parent proxy report scales: An item response theory analysis of the parent proxy report item banks. Qual Life Res . 2012;21(7):1223-1240. doi:10.1007/s11136011-0025-2

27. Varni JW, Thissen D, Stucky BD, et al. PROMIS (r) Parent Proxy Report Scales for children ages 5-7 years: an item response theory analysis of differential item functioning across age groups. Qual Life Res . 23:349-361. doi:10.2307/24726304

28. Varni JW, Burwinkle TM. The PedsQL ${ }^{\mathrm{TM}}$ as a patient-reported outcome in children and adolescents with Attention-Deficit/Hyperactivity Disorder: A population-based study. Health Qual Life Outcomes . 2006;4:26. doi:10.1186/1477-7525-4-26

29. Bjorner JB, Rose M, Gandek B, Stone AA, Junghaenel DU, Ware JE. Method of administration of PROMIS scales did not significantly impact score level, reliability, or validity. J Clin Epidemiol . 2014;67(1):108-113. doi:10.1016/j.jclinepi.2013.07.016

30. Magnus BE, Liu Y, He J, et al. Mode effects between computer self-administration and telephone interviewer-administration of the PROMIS(r) pediatric measures, self- and proxy report. Qual Life Res . 2016;25(7):1655-1665. doi:10.1007/s11136-015-1221-2

31. PROMIS Pediatric and Parent Proxy Profile Scoring Manual .; 2020. doi:10.32388/pvl6lk

32. Irwin DE, Stucky BD, Thissen D, et al. Sampling plan and patient characteristics of the PROMIS pediatrics large-scale survey. Qual Life Res . 2010;19(4):585-594. doi:10.1007/s11136-010-9618-4

33. PROMIS Score Cut Points. https://www.healthmeasures.net/score-and-interpret/interpretscores/promis/promis-score-cut-points. Accessed July 4, 2020.

34. Thissen D, Liu Y, Magnus B, et al. Estimating minimally important difference (MID) in PROMIS pediatric measures using the scale-judgment method. Qual Life Res . 2016;25(1):13-23. doi:10.1007/s11136015-1058-8

35. Mack JW, McFatrich M, Withycombe JS, et al. Agreement Between Child Self-report and CaregiverProxy Report for Symptoms and Functioning of Children Undergoing Cancer Treatment. JAMA Pediatr . August 2020:e202861-e202861. doi:10.1001/jamapediatrics.2020.2861

36. Westmoreland KD, Montgomery ND, Stanley CC, et al. Plasma Epstein-Barr virus DNA for pediatric Burkitt lymphoma diagnosis, prognosis and response assessment in Malawi. Int J cancer . 2017;140(11):25092516. doi:10.1002/ijc.30682 
37. Hinds PS, Wang J, Cheng YI, et al. PROMIS pediatric measures validated in a longitudinal study design in pediatric oncology.Pediatr Blood Cancer . 2019;66(5). doi:10.1002/pbc.27606

38. Knaul FM, Farmer PE, Krakauer EL, et al. Alleviating the access abyss in palliative care and pain relief\&\#x2014;an imperative of universal health coverage: the $<$ em $>$ Lancet $</$ em $>$ Commission report. Lancet . 2018;391(10128):1391-1454. doi:10.1016/S0140-6736(17)32513-8

39. Liben S, Papadatou D, Wolfe J. Paediatric palliative care: challenges and emerging ideas. Lancet . 2008;371(9615):852-864. doi:10.1016/S0140-6736(07)61203-3

40. Weaver MS, Yao AJJ, Renner LA, Harif M, Lam CG. The prioritisation of paediatrics and palliative care in cancer control plans in Africa.Br J Cancer . 2015;112(12):1845-1856. doi:10.1038/bjc.2015.158

41. DeWalt DA, Gross HE, Gipson DS, et al. PROMIS(r) pediatric self-report scales distinguish subgroups of children within and across six common pediatric chronic health conditions. Qual Life Res . 2015;24(9):21952208. doi:10.1007/s11136-015-0953-3

42. DeWalt DA, Thissen D, Stucky BD, et al. PROMIS pediatric peer relationships scale: Development of a peer relationships item bank as part of social health measurement. Heal Psychol . 2013;32(10):1093-1103. doi: $10.1037 / \mathrm{a} 0032670$

43. Stanley CC, Westmoreland KD, Itimu S, et al. Quantifying bias in survival estimates resulting from loss to follow-up among children with lymphoma in Malawi. Pediatr Blood Cancer . 2017;64(6):10.1002/pbc.26370. doi:10.1002/pbc.26370

44. Gopal S, Gross TG. How I treat Burkitt lymphoma in children, adolescents, and young adults in subSaharan Africa. Blood . 2018;132(3):254-263. doi:10.1182/blood-2018-04-844472

45. McGoldrick SM, Mutyaba I, Adams S V., et al. Survival of children with endemic Burkitt lymphoma in a prospective clinical care project in Uganda. Pediatr Blood Cancer . 2019;66(9). doi:10.1002/pbc.27813

46. Yao AJ-J, Moreira C, Traore F, et al. Treatment of Wilms Tumor in Sub-Saharan Africa: Results of the Second French African Pediatric Oncology Group Study. J Glob Oncol . 2019;5(5):1-8. doi:10.1200/jgo.18.00204

47. Patte C, Auperin A, Gerrard M, et al. Results of the randomized international FAB/LMB96 trial for intermediate risk B-cell non-Hodgkin lymphoma in children and adolescents: it is possible to reduce treatment for the early responding patients. Blood . 2007;109(7):2773-2780. doi:10.1182/blood-2006-07-036673

48. Wildes TM, Farrington L, Yeung C, et al. Rituximab is associated with improved survival in Burkitt lymphoma: a retrospective analysis from two US academic medical centers. Ther Adv Hematol . 2014;5(1):312. doi: $10.1177 / 2040620713514682$

49. Broderick JE, May M, Schwartz JE, et al. Patient reported outcomes can improve performance status assessment: a pilot study. J Patient-Reported Outcomes . 2019;3(1):41. doi:10.1186/s41687-019-0136-z

50. Myers RM, Balsamo L, Lu X, et al. A prospective study of anxiety, depression, and behavioral changes in the first year after a diagnosis of childhood acute lymphoblastic leukemia: a report from the Children's Oncology Group. Cancer . 2014;120(9):1417-1425. doi:10.1002/cncr.28578

51. Njuguna F, Mostert S, Slot A, et al. Abandonment of childhood cancer treatment in Western Kenya. Arch Dis Child Educ Pract Ed . 2014;99(7):609-614. doi:10.1136/archdischild-2013-305052

52. Togo B, Traore F, Togo AP, et al. Epidemiologie et pronostic des cancers pediatriques au CHU GabrielToure de Bamako (Mali). Med Sante Trop . 2014;24(1):68-72. doi:10.1684/mst.2014.0291

Figures:

Figure 1. Flow chart for Pediatric PROMIS-25 surveys completed by participants receiving lymphoma care in Lilongwe, Malawi. 
$\mathrm{LTFU}=$ lost to follow-up.

Figure 2. Cox Hazard Ratios and 1-year Kaplan-Meier Overall survival curves for pediatric lymphoma patients at $\mathrm{KCH}$ in Lilongwe, Malawi, stratified by poor physical functioning at diagnosis.

Cohort overall survival at 1-year was 47\% (95\% CI [37-59\%]

Figure 3. Distribution of PROMIS-25 HRQoL domain scores between diagnosis $(n=75)$, active treatment $(n=35)$, and follow-up $(n=24)$ for pediatric lymphoma patients treated in Lilongwe, Malawi.

Higher PROMIS scores for Mobility and Peer Relationships reflect better functioning and relationships; lower symptom scores reflect better symptom experiences.

Figure 4. Trajectories of PROMIS-25 HRQoL domain scores throughout cancer care at KCH in Lilongwe, Malawi among patients who completed surveys at all time points $(n=17)$. Unique patient trajectory indicated by colored line; mean domain score at each timepoint indicated by black dot; mean reference T-score of 50 indicated by black horizontal line. Higher PROMIS scores for Mobility and Peer Relationships reflect better functioning and relationships; lower symptom scores reflect better symptom experiences.

Tables:

Table 1. Characteristics of pediatric lymphoma patients who completed Pediatric PROMIS-25 surveys at diagnosis in Lilongwe, Malawi, stratified by outcome.

Table 2. PROMIS-25 Pediatric Health-related Quality of Life domain scores and Pain Intensity throughout pediatric lymphoma treatment in Lilongwe, Malawi.

$\Delta=$ Difference in mean domain score between timepoints.

Supplemental Material (for Review):

Supplemental Table 1. Sensitivity Analysis Patient Self-Reported (excluding parent proxyreported surveys) of PROMIS-25 Pediatric Health-related Quality of Life domain scores and Pain Intensity throughout pediatric lymphoma treatment in Lilongwe, Malawi.

Supplemental Figure 1. One-year Kaplan-Meier Overall survival curves for pediatric lymphoma patients at KCH in Lilongwe, Malawi, stratified by patient-reported PROMIS Mobility Domain Score $<40$ at diagnosis.

$\mathrm{HR}=2.05,95 \%$ CI $[0.73,5.73], \mathrm{p}=0.17$.

Hosted file

Table 1.pdf available at https://authorea.com/users/374614/articles/492088-pediatriclymphoma-patients-in-malawi-present-with-poor-health-related-quality-of-life-atdiagnosis-and-improve-throughout-treatment-and-follow-up-across-all-pediatric-promis25-domains

Hosted file

Table 2.pdf available at https://authorea.com/users/374614/articles/492088-pediatriclymphoma-patients-in-malawi-present-with-poor-health-related-quality-of-life-atdiagnosis-and-improve-throughout-treatment-and-follow-up-across-all-pediatric-promis25-domains 

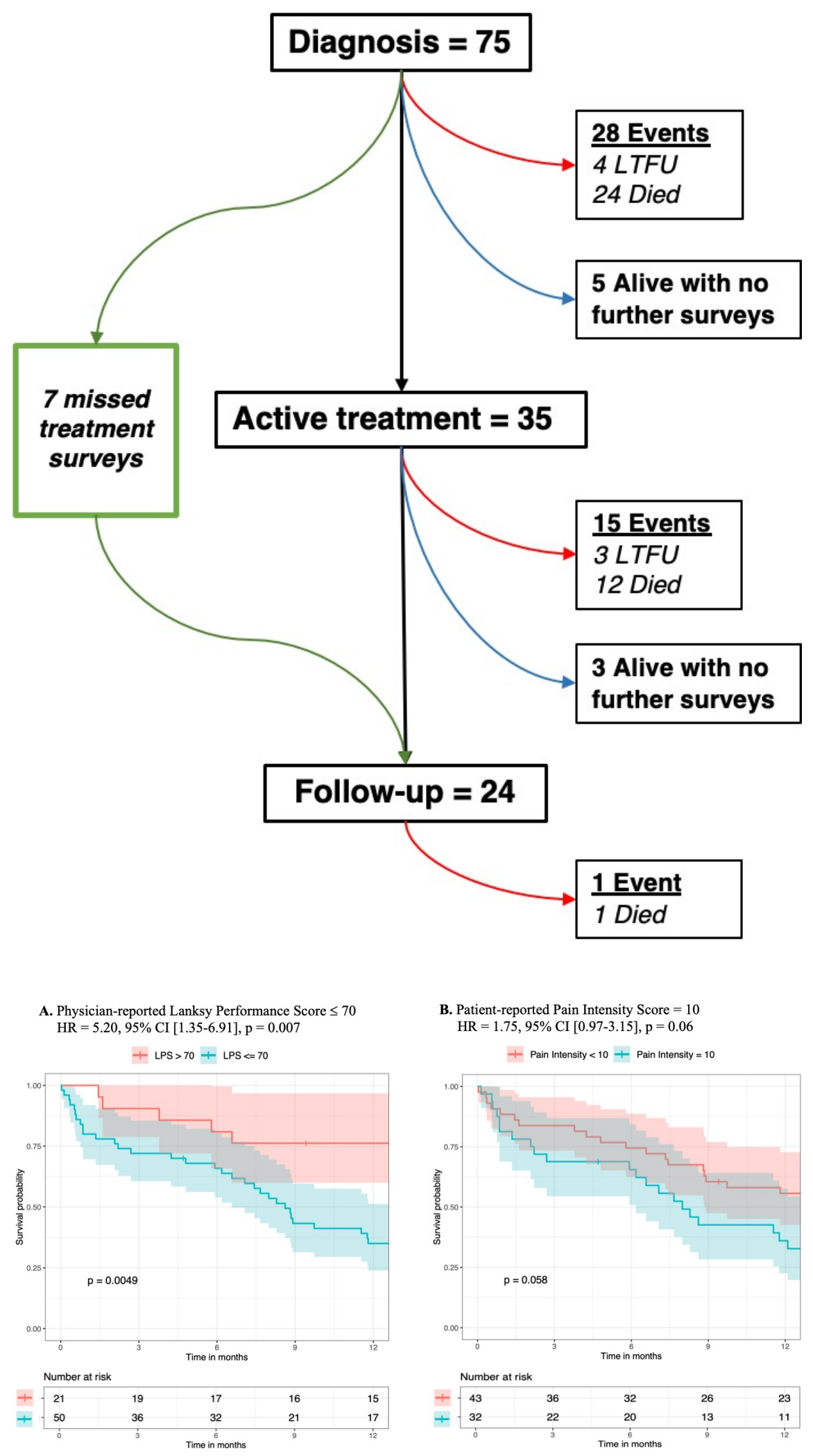

B. Patient-reported Pain Intensity Score $=10$ $\mathrm{HR}=1.75,95 \%$ CI [0.97-3.15], $\mathrm{p}=0.06$

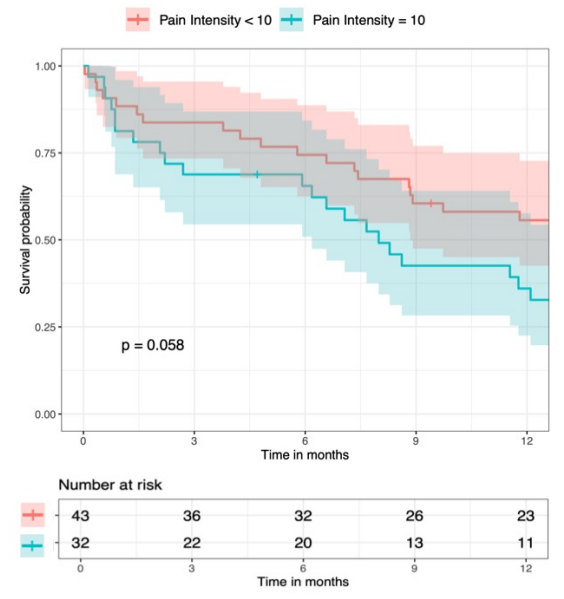




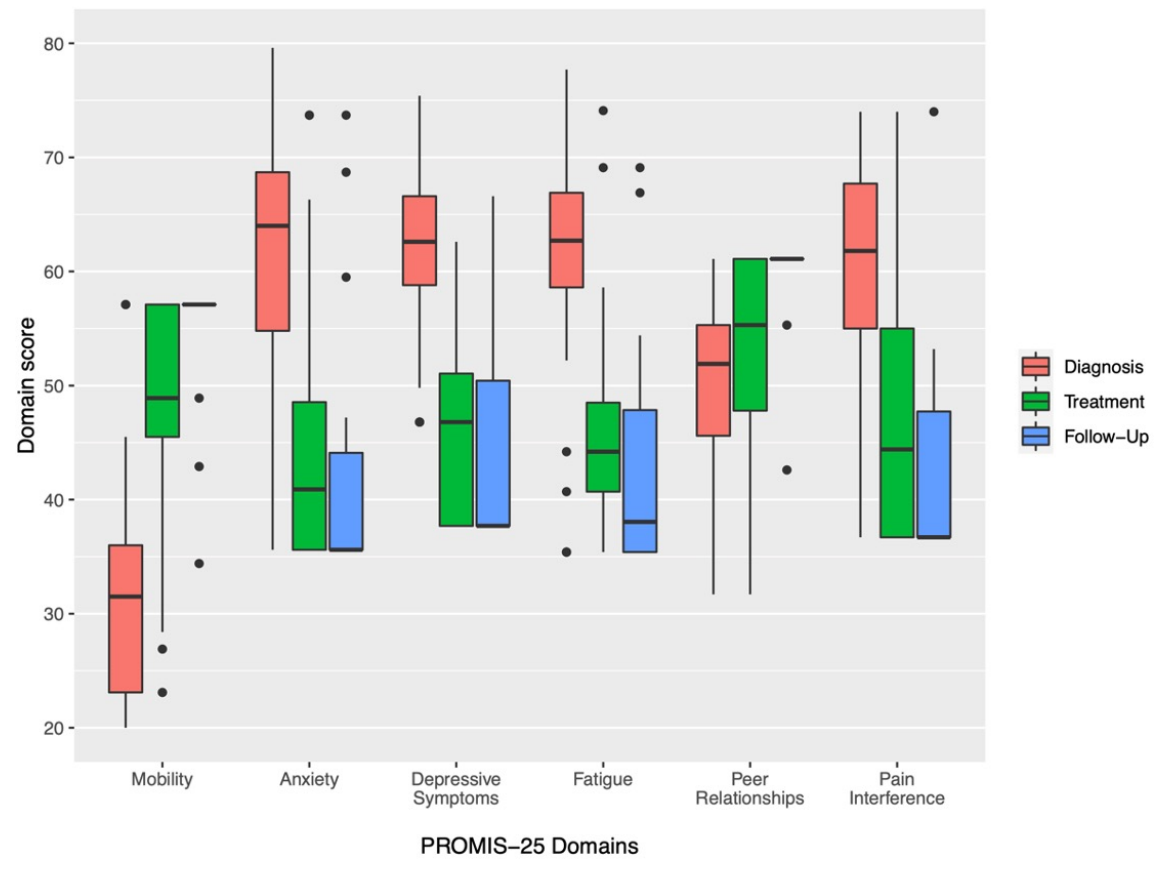



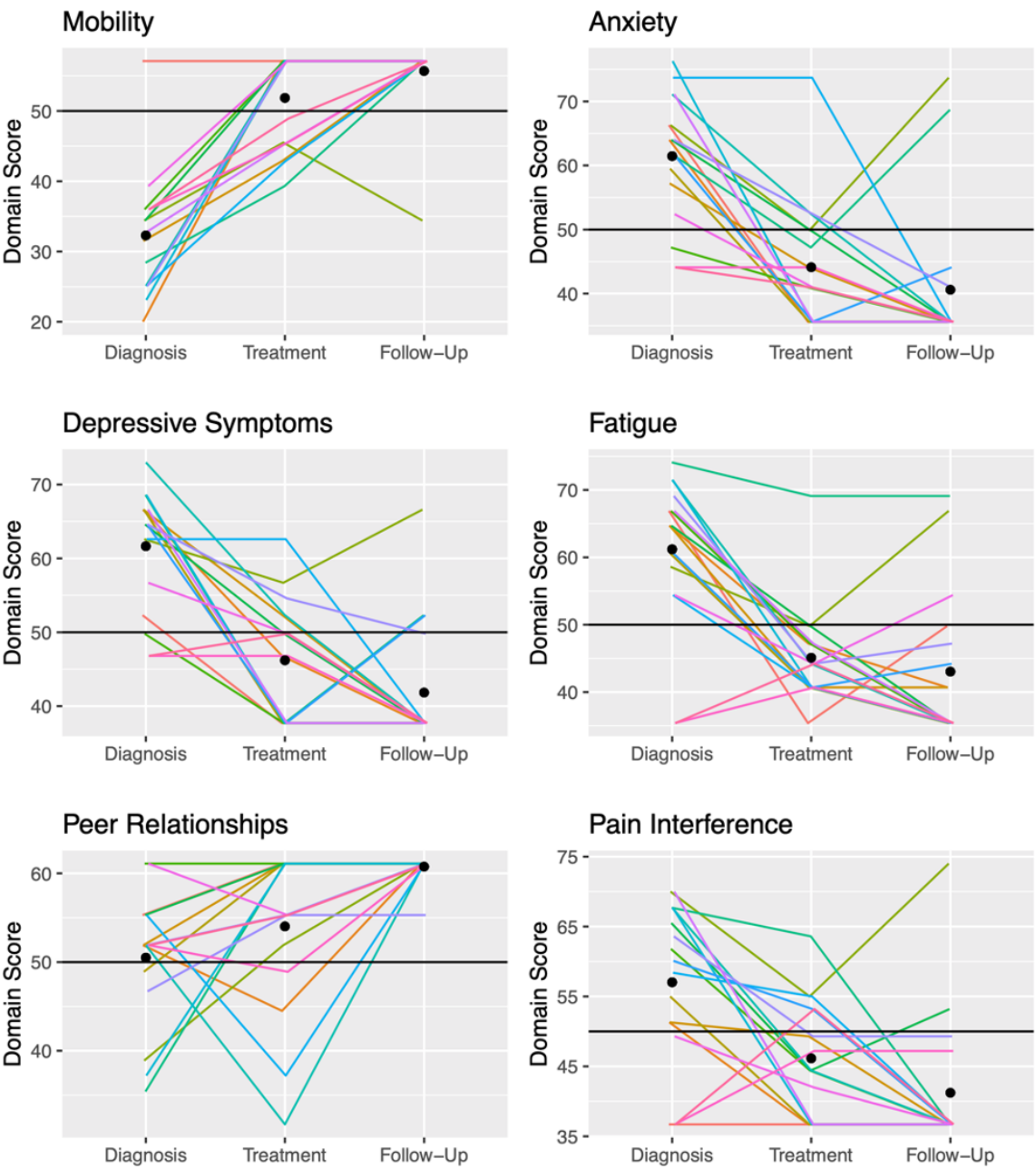


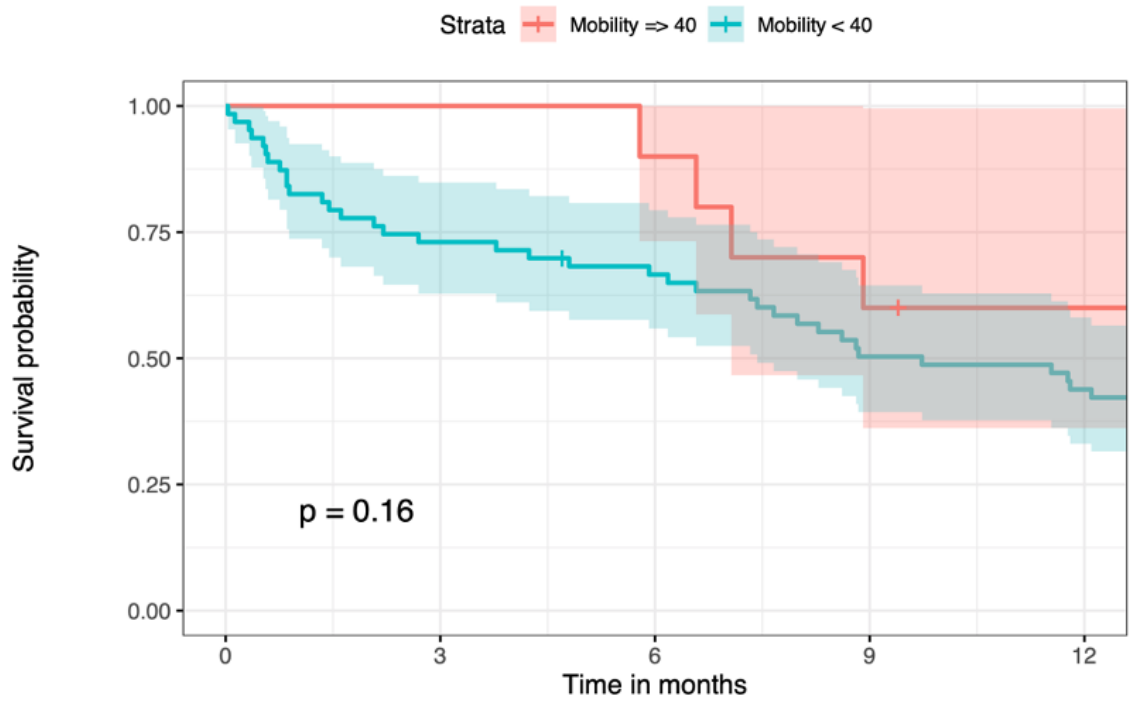

\begin{tabular}{|c|c|c|c|c|c|}
\hline \multirow{4}{*}{ 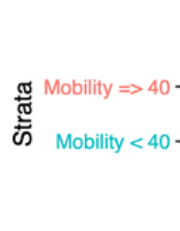 } & \multicolumn{5}{|c|}{ Number at risk } \\
\hline & 10 & 10 & 9 & 6 & 5 \\
\hline & 63 & 46 & 41 & 31 & 27 \\
\hline & 0 & 3 & $\begin{array}{c}6 \\
\text { in } \mathrm{m}\end{array}$ & 9 & 12 \\
\hline
\end{tabular}

